\title{
THE COMPLETE EXISTENTIAL THEORY OF THE WHITEHEAD-HUNTINGTON SET OF POSTULATES FOR THE ALGEBRA OF LOGIC*
}

\author{
BY \\ A. H. DIAMOND
}

1. Introduction. Consider any set of postulates, say, for the sake of concreteness, $\mathbf{P}_{\mathbf{1}}, \mathbf{P}_{\mathbf{2}}, \mathbf{P}_{\mathbf{8}}$. Any interpretation of the undefined ideas of $\mathbf{P}_{\mathbf{1}}, \mathbf{P}_{\mathbf{2}}$, $\mathrm{P}_{3}$ constitutes a concrete system $S$ which either satisfies or does not satisfy some or all of the postulates. The system $S$ will then have with respect to $\mathrm{P}_{1}, \mathrm{P}_{2}, \mathrm{P}_{3}$ one of the $2^{3}$ characters $( \pm \pm \pm)$, where a " + " sign in the $i$ th place denotes that postulate $P_{i}$ is satisfied and a " -" sign that $P_{i}$ is not satisfied. The complete existential theory of the postulate-set $\mathrm{P}_{1}, \mathrm{P}_{2}, \mathrm{P}_{3}$ consists in determining for every one of the $2^{3}$ characters $( \pm \pm \pm)$ whether or not there exists a concrete system $S$ corresponding to that character. $\dagger$ The object of this paper is to establish the complete existential theory of the WhiteheadHuntingtor: set of ten postulates for the algebra of logic, expressed in terms of logical addition and logical multiplication. $\neq$ This is, perhaps, the most "natural" and most elegant set of postulates for the Boole-Schröder algebra of logic.

The complete existential theory of a set of postulates includes the solution of the problem of determining whether or not the postulates of the set (or of any of its sub-sets) are completely independent, i.e., whether or not any of the postulates of the set (or sub-set) or their denials can be derived from any of the other postulates or their denials. Thus the present discussion will show whether or not the postulates of the Whitehead-Huntington set (or any of its sub-sets) are completely independent.

The present theory has the following distinctive characteristics. (1) The number of postulates involved is 10 , and so requires, for the establishment of the complete existential theory, $2^{10}$ propositions of existence and nonexistence. This number is far greater than the number of propositions which constitute any complete existential theory hitherto published.§ (2) The con-

* Presented to the Society, March 18, 1933; received by the editors March 18, 1933.

† Professor E. H. Moore first proposed the problem of the complete existential theory of a set of postulates. See his Introduction to a Form of General Analysis, New Haven Colloquium, Yale University Press, p. 82.

† See E. V. Huntington, these Transactions, vol. 5 (1904), pp. 288-309.

$\S$ The largest number hitherto published is $2^{6}=64$. See B. A. Bernstein, The complete existential theory of Huruitz's postulates for abelian groups and fields, Bulletin of the American Mathematical 
crete systems employed are all in the modular form devised by Professor B. A. Bernstein.* This form permits the listing of the large number of systems involved with a conciseness and a simplicity not existing in the proof-systems employed before the development of the modular theory in question. (3) The number of systems constituting the propositions of existence is 325 . This number is far larger than any previous number of proof-systems employed in connection with a set of postulates. Further, the systems relate to postulates expressing laws found in many other mathematical theories. These systems thus provide a large store of possible proof-systems for many important postulate-sets. (4) The systems employed are all algebras of not more than three elements. This adds to the value of the systems as a source of possible proof-systems to be used in other postulate-sets.

I begin with the listing of the Whitehead-Huntington postulates.

2. The Whitehead-Huntington postulates. The Whitehead-Huntington set of postulates for the algebra of logic leave undefined a class $K$, and two binary operations,$+ \times$, and are the ten propositions following. $\dagger$

Ia. $a+b$ is in $K$ whenever $a$ and $b$ are in $K$.

Ib. $a b$ is in $K$ whenever $a$ and $b$ are in $K$.

IIa. There is an element $Z$ such that $a+Z=a$ for every element $a$.

IIb. There is an element $U$ such that $a U=a$ for every element $a$.

IIIa. $a+b=b+a$ whenever $a, b, a+b$, and $b+a$ are in $K$.

IIIb. $a b=b a$ whenever $a, b, a b$, and $b a$ are in $K$.

IVa. $a+b c=(a+b)(a+c)$ whenever $a, b, c, a+b, a+c, b c, a+b c$, and $(a+b)(a+c)$ are in $K$.

IVb. $a(b+c)=a b+a c$ whenever $a, b, c, a b, a c, b+c, a(b+c)$, and $a b+a c$ are in $K$.

V. If the elements $Z$ and $U$ in postulates IIa and IIb exist and are unique, then for every element $a$ there is an element $a^{\prime}$ such that $a+a^{\prime}=U$ and $a a^{\prime}=Z$.

VI. There are at least two elements, $x$ and $y$, in $K$ such that $x \neq y$.

Society, vol. 28 (1922), p. $397.2^{5}=32$ propositions occur in two other papers. See Paul Henle, The independence of the postulates of logic, Bulletin of the American Mathematical Society, vol. 38 (1932), p. 409. See also J. S. Taylor, Sheffer's set of five postulates for Boolean algebras in terms of the operation "rejection" made completely independent, Bulletin of the American Mathematical Society, vol. 26 (1920), p. 449.

* See B. A. Bernstein, Modular representations of finite algebras, Proceedings of the International Mathematical Congress, Toronto, 1924,p. 207. See also B. A. Bernstein and Nemo Debely, A practical method for the modular representation of finite operations and relations, Bulletin of the American Mathematical Society, vol. 38 (1932), p. 110.

† The original wording of the postulates is retained except that $K$ replaces Huntington's "class", the circles around the operations + and $X$ are omitted, and the original $\wedge, \vee$, and $d$ are replaced by $Z, U$ and $a^{\prime}$ respectively. 
If these postulates be denoted by $a_{1}, a_{2}, \cdots, a_{10}$ and their denials by $a_{1}^{\prime}, a_{2}^{\prime}, \cdots, a_{10}^{\prime}$, then the propositions $a_{1}, a_{2}, \cdots, a_{10}, a_{1}^{\prime}, a_{2}^{\prime}, \cdots, a_{10}^{\prime}$ divide the universe of discourse of these propositions into $2^{10}=1024$ compartments represented by the logical products $a_{1} a_{2} \cdots a_{10}, a_{1} a_{2} \cdots a_{10}^{\prime}$, $\cdots, a_{1}^{\prime} a_{2}^{\prime} \cdots a_{10}^{\prime} .^{*}$ Only 325 of these compartments are actually represented in the universe; the remaining 699 are empty because of the relations of implication subsisting among the propositions $a_{1}, a_{2}, \cdots, a_{10}, a_{1}^{\prime}, a_{2}^{\prime}$, $\cdots, a_{10}^{\prime}$.

3. Propositions of non-existence. Of the $2^{10}$ propositions constituting the complete existential theory of our postulates, $2^{10}-325=699$ are propositions of non-existence. These non-existence propositions, together with reasons establishing them, are given by propositions $\mathrm{A}-\mathrm{F}$ following.

A. There exist no systems for the characters ( \pm \pm \pm \pm \pm \pm \pm \pm \pm-$)$ except ( \pm \pm \pm \pm+++++-$)$.

For, if postulate VI is denied by a system $S$, then $K$ has just one element or none. If $K$ has no elements, $S$ satisfies postulates IIIa, IIIb, IVa, IVb, and $\mathrm{V}$ vacuously. If $K$ has just one element, there are four cases to consider. (1) If $S$ satisfies both postulates Ia and Ib, then $S$ satisfies postulates IIIa, IIIb, IVa, IVb, and V non-vacuously. (2) If $S$ denies both postulates Ia and Ib, then $S$ satisfies postulates IIIa, IIIb, IVa, IVb, and V vacuously. (3) If $S$ satisfies postulate Ia and denies postulate Ib, then $S$ satisfies postulate IIIa non-vacuously and postulates IIIb, IVa, IVb, and V vacuously. (4) If $S$ denies postulate Ia and satisfies postulate Ib, then $S$ satisfies IIIb non-vacuously and postulates IIIa, IVa, IVb, and V vacuously.

Proposition A accounts for 496 characters.

B. There exist no systems for the characters $(- \pm+ \pm \pm \pm \pm \pm \pm-)$.

For, if postulate VI is denied, postulate IIa will be satisfied if and only if $K$ has just one element and $S$ satisfies postulate Ia.

Proposition B accounts for 4 characters not already accounted for by proposition A, namely $(- \pm+ \pm+++++-)$.

C. There exist no systems for the characters $( \pm- \pm+ \pm \pm \pm \pm \pm-)$.

For, if postulate VI is denied, postulate IIb will be satisfied if and only if $K$ has just one element and $S$ satisfies postulate Ib.

Proposition $\mathrm{C}$ accounts for 3 characters not already accounted for by propositions $\mathrm{A}$ and $\mathrm{B},( \pm--++++++-)$ and $(+-+++++++-)$.

D. There exist no systems for the characters $(+++- \pm \pm \pm \pm \pm-)$ and $(++-+ \pm \pm \pm \pm \pm-)$.

* See E. V. Huntington, these Transactions, vol. 26 (1924), p. 277. 
For, if postulate VI is denied, there are two ways in which postulates Ia and Ib may both be satisfied. (1) If $K$ has no elements, postulates Ia and Ib are satisfied vacuously. (2) If $K$ has just one element, postulates Ia and Ib may both be satisfied non-vacuously. In case (1) both postulates IIa and IIb are denied. In case (2) both postulates IIa and IIb are satisfied.

Proposition D accounts for 2 characters not already accounted for by propositions $\mathrm{A}, \mathrm{B}$, and $\mathrm{C}$, namely $(+++-+++++-)$ and $(++-++$ $++++-)$.

E. There exist no systems for the characters ( +-- \pm \pm \pm \pm \pm-$)$ and $(-+-- \pm \pm \pm \pm \pm-)$.

For, if postulate VI is denied, there are two ways in which postulates IIa and IIb may both be denied. (1) If $K$ has no elements, postulates IIa and IIb are denied non-vacuously and postulates Ia and Ib are satisfied vacuously. (2) If $K$ has just one element, postulates IIa and IIb are both denied if and only if postulates Ia and $\mathrm{Ib}$ are both denied.

Proposition $\mathrm{E}$ accounts for 2 characters not already accounted for by propositions $\mathrm{A}, \mathrm{B}, \mathrm{C}$, and $\mathrm{D}$, namely $(+---+++++-)$ and $(-+--$ $+++++-)$.

F. There exist no systems for the characters ( \pm \pm+- \pm \pm \pm \pm- \pm ), ( \pm \pm-+ \pm \pm \pm \pm- \pm ), and ( \pm \pm-- \pm \pm \pm \pm- \pm ).

For, if postulate IIa or IIb or both are denied, then postulate $\mathrm{V}$ is satisfied vacuously.

Proposition $\mathrm{F}$ accounts for 192 characters not already accounted for by propositions $\mathrm{A}, \mathrm{B}, \mathrm{C}, \mathrm{D}$, and $\mathrm{E}$, namely $( \pm \pm+- \pm \pm \pm \pm-+),( \pm \pm$ $-+ \pm \pm \pm \pm-+)$ and $( \pm \pm-- \pm \pm \pm \pm-+$ ).

4. Propositions of existence. The 325 propositions of existence for our postulates are given by the tables $\mathrm{A}$ and $\mathrm{B}$ below. In these tables all the systems are arithmetic systems, the elements being the numbers 0,1 , and 2 . The notations used are those employed by Professor Bernstein in the second of the papers cited in the first footnote on page 941, except that, for the sake of saving space, certain abbreviations are resorted to. Let $f(a, b)$ be any polynomial expression in $a$ and $b$, where $a$ and $b$ are any of the numbers $0,1, \cdots$, $p-1$. Then $(f(a, b))_{p}$ will denote the least positive residue modulo $p$ obtained from $f(a, b)$ by rejecting multiples of $p$. The operations + and $\times$ are to be interpreted as the operations of ordinary arithmetic when they occur in the modular expressions, otherwise they are to be interpreted as logical addition and logical multiplication. Thus for $a=1, b=1$, we have $\left(a^{2}+a b+b+2\right)_{3}=2$. $[a, b ; m, n]_{p}$ will denote a function $(f(a, b))_{p}$ such that $(f(a, b))_{p}=0$ or 1 according as the equalities $a=m, b=n$ do or do not both hold. The results 
obtained by Professor Bernstein enable us to write down the expression for $[a, b ; m, n]_{p}$. Indeed, $[a, b ; m, n]_{p}=\left(1-\left\{1-(a-m)^{p-1}\right\}\left\{1-(b-n)^{p-1}\right\}\right)_{p}$. Thus $[a, b ; 1,2]_{3}=\left(1-\left\{1-(a-1)^{2}\right\}\left\{1-(b-2)^{2}\right\}\right)_{3}=\left(2 a^{2} b^{2}+a^{2} b+2 a b^{2}\right.$ $+a b+1)_{3}$.

For the sake of simplicity, the 325 systems will be divided into two groups, $\mathrm{A}$ and $\mathrm{B}$, according as $K$ does not have or does have more than one element. Table A gives systems for which $K$ does not have more than one element; table $\mathrm{B}$ gives systems for which $K$ does have more than one element. To save space in table $\mathrm{B}$, instead of writing out the elements of $K$ as in table A, the subscript $p$ in $(f(a, b))_{p}$ will indicate that the elements of $K$ are $0,1, \cdots$, $p-1$. In both tables, the characters will be written without parentheses.

The duality of the postulates with respect to the operations + and $X$ makes it possible to reduce the number of systems in table B from 320 to 172. Every system $S$ has a dual system obtained from $S$ by interchanging the definitions of + and $\times$. The character corresponding to the dual of a system $S$ is determined as follows. Consider the character $C$ corresponding to $S$. $C$ consists of "+" and "-" signs arranged in a certain order. The first 8 signs occur in pairs corresponding to dual postulates. Interchange in $C$ the signs in each pair. The character thus obtained is the character corresponding to the dual of $S$.

Table A

\begin{tabular}{l|c|c|c|c}
\hline \hline \multicolumn{1}{c|}{ No. } & Character & $K$ & $a+b$ & $a b$ \\
\hline (i) & ----+++++- & 0 & $0 / 0$ & $0 / 0$ \\
(ii) & -+-++++++- & 0 & $0 / 0$ & 0 \\
(iii) & +-+-+++++- & 0 & 0 & $0 / 0$ \\
(iv) & ++--+++++- & Null & - & - \\
(v) & +++++++++- & 0 & 0 & 0 \\
\hline
\end{tabular}

TABLE B

\begin{tabular}{|c|c|c|c|}
\hline No. & $\begin{array}{c}\text { Character } \\
\text { Ia Ib IIa IIb IIIa IIIb IVa IVb V VI }\end{array}$ & $a+b$ & $a b$ \\
\hline $\begin{array}{l}1 \\
2 \\
3 \\
4 \\
5 \\
6 \\
7 \\
8 \\
8\end{array}$ & 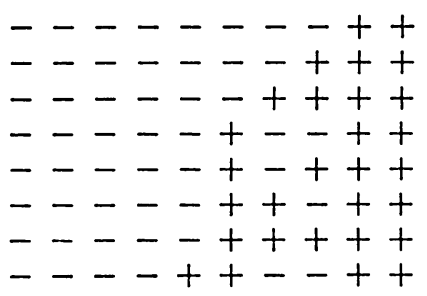 & 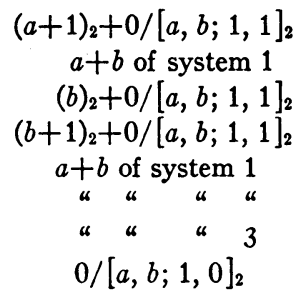 & $\begin{array}{c}(a b+b)_{2}+0 /[a, b ; 0,0]_{2} \\
(a b+a)_{2}+0 /[a, b ; 0,0]_{2} \\
a+b \text { of this system } \\
0 /[a, b ; 1,1]_{2} \\
(a b+1)_{2}+0 /[a, b ; 0,0]_{2} \\
a b \text { of system } 4 \\
a * a \\
1+0 /[a, b ; 1,1]_{2}\end{array}$ \\
\hline
\end{tabular}


TABLE B (Continued)

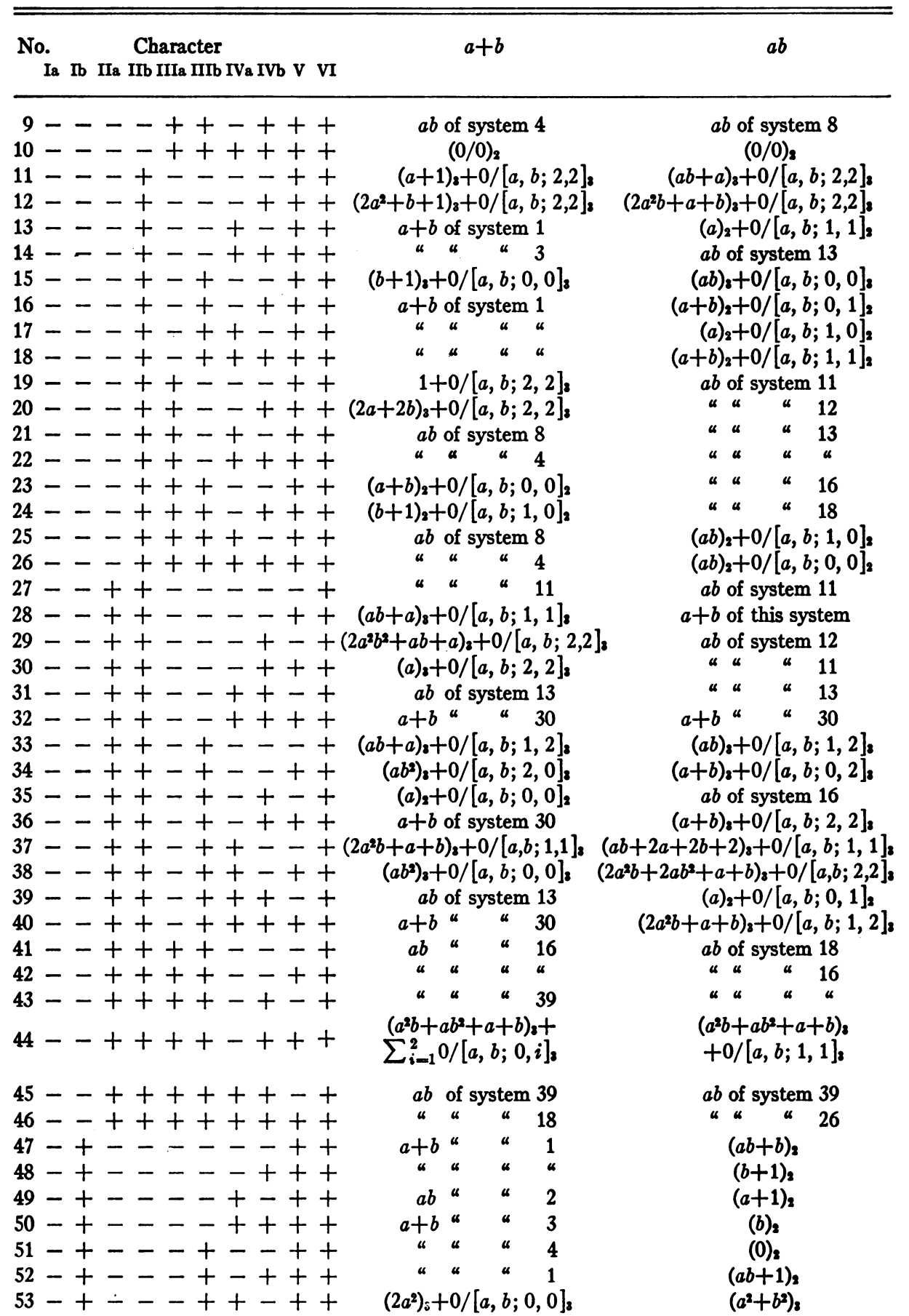


TABLE B (Continued)

\begin{tabular}{llcl}
\hline No. $\quad$ Character & $a+b$ & $a b$ \\
Ia Ib IIa IIb IIIa IIIb IVa IVb V VI & &
\end{tabular}

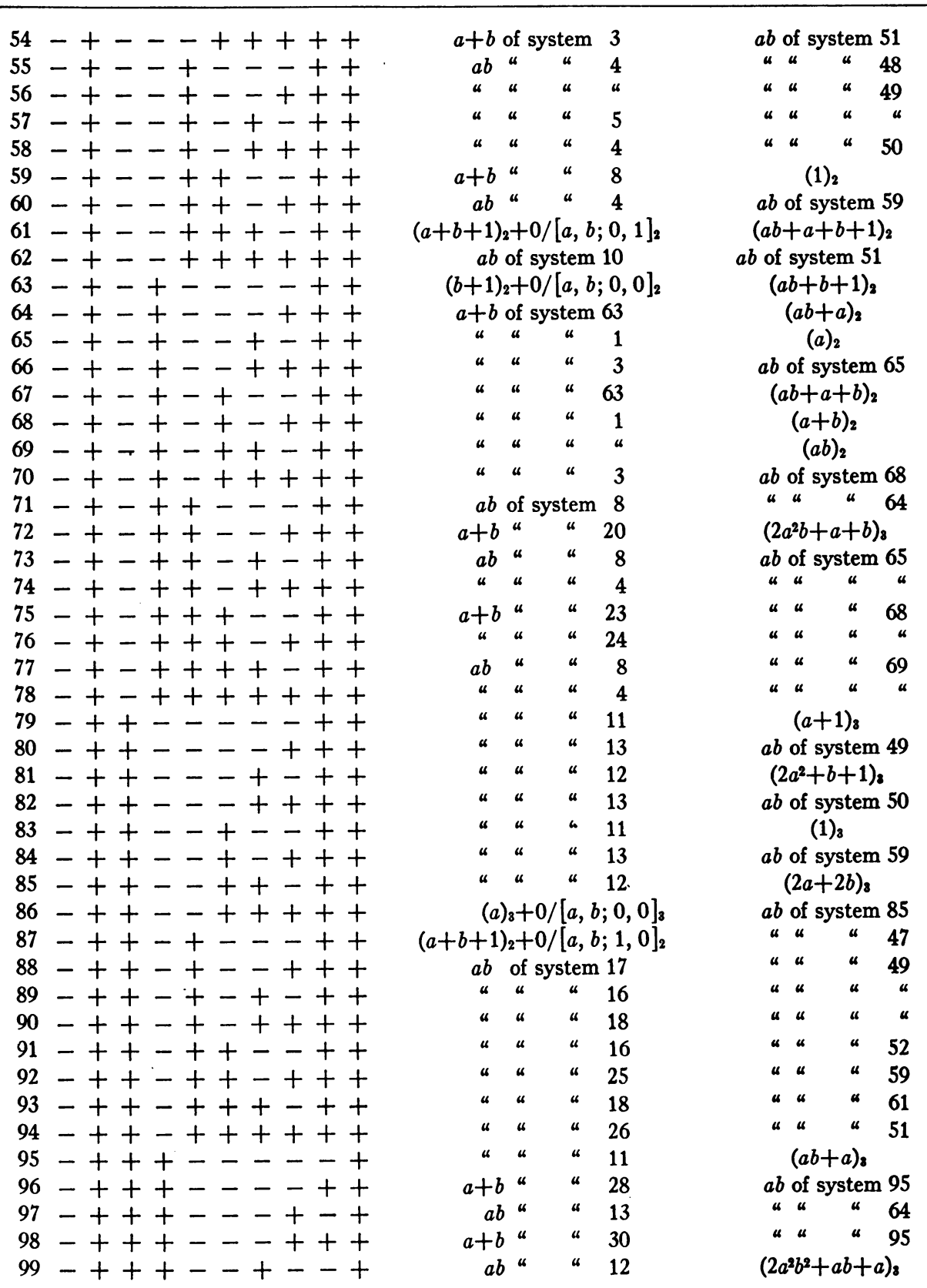


TABLE A (Continued)

No. Character $\quad a+b \quad a b$

Ia Ib IIa IIb IIIa IIIb IVa IVb V VI

$100-+++--+-+$

$101-+++--++-+$

$102-+++--+++$

$103-+++-+---+$

$104-+++-+--++$

$105-+++-+-+-+$

$106-+++-+-+++$

$107-+++-++--+$

$108-+++-++-++$

$109-+++-t++-t$

$110-+++-+++++$

$111-++++---+$

$112-++++---++$

$113-++++--+-+$

$114-++++--++t$

$115-++++-+--+$

$116-++++-+-++$

$117-++++-++-+$

$118-++++-++++$

$119-+++++--+$

$120-+++++--+$

$121-t+t++-+-t$

$122-+++++-+++$

$123-++++++--+$

$124-++++++-++$

$125-t+t++++-t$

$126-++t++t++t$

$127++-----+$

$128++----+++$

$129++----+++$

$130++---+--++$

$131++---+-++$

$132++---++-+$

$133++---++++$

$134++--++--++$

$135++--++-+++$

$136++--+++++$

$137++-+---++$

$138++-+---+++$

$139++-+--+-++$

$140++-+--++++$

$141++-+-+--++$

$142++-+-+-+++$

$143++-+-++-++$

$144++-+-+++++$

$145++-++--++$

\begin{tabular}{|c|c|c|c|}
\hline$a b$ & \multicolumn{2}{|c|}{ of system } & 11 \\
\hline " & “ & " & 12 \\
\hline 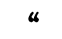 & u & " & 13 \\
\hline$a+b$ & “ & « & 33 \\
\hline$\omega$ & u & " & 34 \\
\hline$a b$ & “ & $\boldsymbol{\omega}$ & 3 \\
\hline$a+b$ & 山 & u & 30 \\
\hline$\mu$ & a & w & 37 \\
\hline u & « & $\boldsymbol{\omega}$ & 8 \\
\hline$a b$ & $\omega$ & $\omega$ & 3 \\
\hline$a+b$ & " & « & 30 \\
\hline$a b$ & " & " & 16 \\
\hline u & “ & u & \\
\hline u & 山 & u & 39 \\
\hline " & u & $\omega$ & 38 \\
\hline u & $\boldsymbol{u}$ & u & 18 \\
\hline a & « & $\alpha$ & 16 \\
\hline$\omega$ & $\boldsymbol{u}$ & u & 39 \\
\hline $\boldsymbol{u}$ & u & u & \\
\hline$\mu$ & « & $\omega$ & \\
\hline$\omega$ & " & $\omega$ & \\
\hline u & " & u & \\
\hline$a+b$ & 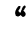 & “ & \\
\hline
\end{tabular}

$(a b)_{3}+0 /[a, b ; 2,2]_{3}$

$a b$ of system 18

« " $\quad 39$

« $4 \quad$ a 18

a $4 \quad$ แ 49

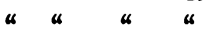

« 4 แ 50

$4 \quad 4 \quad 48$

$\left(2 a^{2}\right)_{8}$

$a+b$ of system 131

$a b$ \& $\quad$ \& 50

a $4 \quad 4 \quad 53$

4 $4 \quad$ a 51

a $4 \quad$ a 48

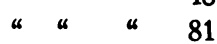

4 4 แ 49

" « 450

a « a 48

a 4 a 4

“ « $4 \quad 49$

4 « a 47

« $4 \quad$ a 59 $a b$

(a)s

$a b$ of system 72

$(a b)_{3}$

$(a+b)_{3}$

$a b$ of system 68

" " " 104

$(a b+2 a+2 b+2)_{3}$

$\left(2 a b^{2}+2 a^{2} b+a+b\right)_{3}$

$a b$ of system 69

$\left(2 a^{2} b^{2}+2 a^{2} b+2 a b^{2}+a b\right)_{3}$

$a b$ of system 63

$\begin{array}{cccc}\omega & \omega & \omega & 64 \\ \omega & u & \omega & 4\end{array}$

$\left(a b^{2}\right)_{3}$

$a b$ of system 63

a « « 65

4 « a 63

* * แ 65

$(a+b+1)_{2}$

$a b$ of system 68

4 a 4 u

$\left(a^{2} b+a b^{2}+a+b\right)_{3}$

$a b$ of system 104

a a a 119

* a $\quad$ a 69

« « $4 \quad 48$

« « $4 ~ 49$

« « $4 ~ 48$

« « 450

« « 451

(0)

$a b$ of system 53

a * a 51

* * 459

* * a 131

a 4 a 51

4 « $4 ~ 64$

* * 472

a * a 65

a a a

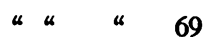

« « $4 ~ 68$

4 4 469

a " 4 "

« « « 64 
TABLE B (Continued)

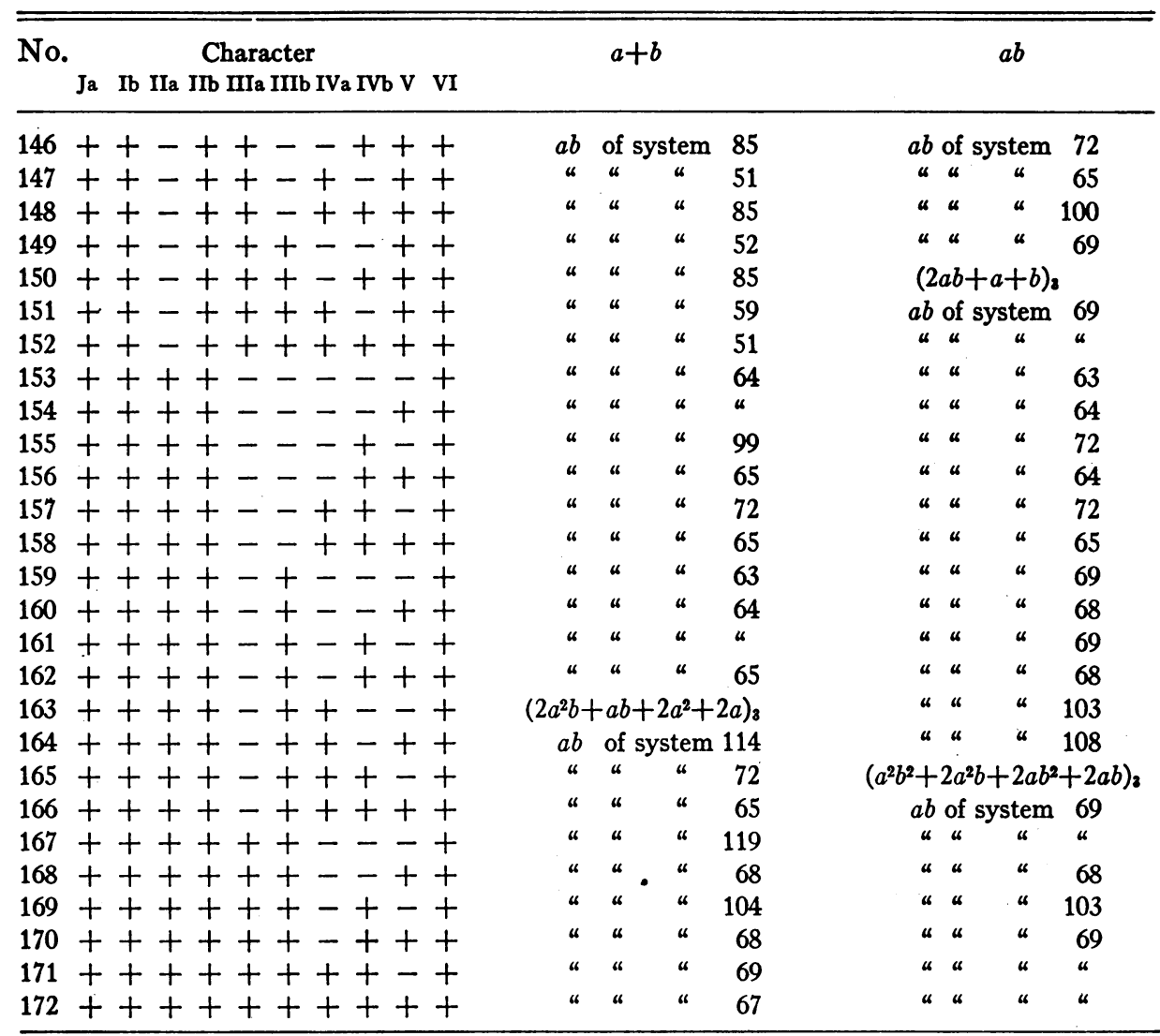

5. Remarks on the systems. In the proof-systems denying postulate Ia, the elements $a$ and $b$ for which $a+b$ does not exist are respectively the values of $m$ and $n$ which occur in the symbol $[a, b ; m, n]_{p}$. Similarly, for postulate Ib.

The verifications will often be more easily effected if tables are constructed corresponding to the modular expressions in question.

UNIVERSITY OF CaITfORNIA, Berkeley, Calit. 